\title{
EVOLUTION DES SPOROCYSTES DE SCHISTOSOMA BOVIS APRẼS TRANSPLANTATION MICROCHIRURGICALE CHEZ BULINUS TRUNCATUS
}

\author{
J. JOURDANE, A. MOUAHID et R. TOUASSEM*
}

RÉSUMÉ. Les transplantations microchirurgicales de sporocystes fils de Schistosoma bovis chez des Bulinus truncatus sains induisent une dédifférenciation des sporocystes transplantés et leur différenciation en sporocystes sporocystogènes producteurs d'une génération additionnelle de sporocystes fils. Ceux-ci colonisent la glande digestive dans sa totalité et produisent des cercaires infestantes.

Durant toute la parasitose une fraction des sporocystes fils issus des sporocystes transplantés persiste dans la zone céphalo-pédieuse et est à son tour le siège d'une sporocystogenèse active. Les données concernant la dynamique de la production cercarienne sont au moins en partie en relation avec la dynamique intramolluscale des populations de sporocystes.

Le succès des transplantations microchirurgicales de sporocystes de $S$. bovis autorise la perspective d'un clonage de cette espèce.

\section{Evolution of the sporocysts of Schistosoma bovis after microsurgical trans- plantation into Bulinus truncatus}

SUMMARY. Microsurgical transplantations of Schistosoma bovis daughter sporocysts to uninfected Bulinus truncatus leads to a dedifferenciation of transplanted sporocysts producing an additional generation of daughter sporocysts. These ones settle into the whole digestive gland and produce infective cercariae.

For all the parasitosis, a part of the daughter sporocysts born from the transplanted ones, remains in the head-foot region and are in their turn responsible for an active sporocystogeny.

Data demonstrate that the cercarial production dynamics is at least in part in relation with the intramolluscal dynamics of sporocyst populations.

Success of microsurgical transplantation of $S$. bovis sporocysts permits cloning perspective of this species.

La réplication des sporocystes, c'est-à-dire leur potentialité d'arrêter la production de cercaires pour s'orienter dans un processus de sporocystogenèse a été mise en évidence au cours de la multiplication végétative de Schistosoma mansoni (Jourdane et al., 1980) et de S. haematobium (Kechemir et Théron, 1980). Cette propriété a permis le clonage de ces espèces par transplantation microchirurgicale de sporocystes

* Département de Biologie Animale, Université (Directeur : P. C. Combes), Avenue de Villeneuve, F 66025 Perpignan Cedex.

Accepté le 19 décembre 1983. 
de Mollusque à Mollusque (Jourdane et Théron, $1980 \mathrm{chez}$ S. mansoni et Jourdane et al., 1981 chez S. haematobium). Combes et al. (1983) ont récemment démontré que le processus de réplication intervient aussi comme mode habituel de reproduction dans le cycle de Schistosoma bovis aussi bien chez Bulinus truncatus que chez Planorbarius metidjensis. L'existence de cette potentialité chez $S$. bovis nous a conduit à étendre les recherches sur le clonage des Schistosomes à cette dernière espèce ; nous présentons ici les premiers résultats obtenus dans cette voie.

\section{Matériel et méthodes}

Le matériel d'étude sur lequel nous avons travaillé est représenté par le couple hôte-parasite : Schistosoma bovis d'origine soudanaise et Bulinus truncatus d'origine tunisienne.

La technique opératoire utilisée s'inspire en tous points de celle mise au point par Jourdane et al. (1981) pour Bulinus truncatus. Les greffons comprennent 2 à 4 sporocystes fils prélevés chez des Mollusques donneurs parasités depuis 90 jours. Les transplantations ont été réalisées sur un lot de 20 Bulins mesurant 9 à $10 \mathrm{~mm}$ de longueur.

Des études histologiques sur coupes sériées de Mollusques ont été faites à différents stades $(8,18,79$ et 118 jours) pour apprécier l'évolution du greffon et le développement de la parasitose ; les coupes ont été colorées à l'azan de Heindenheim.

L'infectivité des cercaires a été testée après infestation de Souris de laboratoire dans 50 cc d'eau avec 250 cercaires et récupération des adultes par perfusion directe (Duwall et Dewitt, 1967) 5 semaines après l'infestation.

Dès le début de l'émission cercarienne, les Mollusques positifs sont répartis individuellement dans des cristallisoirs contenant environ $150 \mathrm{ml}$ d'eau. La production a été suivie quotidiennement pendant 12 semaines. La filtration et le dénombrement des cercaires ont été réalisés suivant la méthode de Théron (1982).

\section{Résultats}

\section{Évolution histologique du greffon}

$\mathrm{a}-8$ jours

A ce stade, le greffon est localisé dans le système lacunaire du sinus céphalopédieux du Mollusque. Le contenu des sporocystes est le siège d'une dégénérescence touchant les cercaires mûres. Les cercaires sorties des sporocystes et égarées dans les différents organes du Mollusque sont encapsulées et détruites. Il n'y a pas à proprement parler d'adhésion véritable entre le greffon et les tissus de l'hôte; le même phénomène a été observé pour $S$. haematobium chez B. truncatus (Jourdane et al., 1981) et $P$. metidjensis (Kechemir et Combes, 1982). 
b -18 jours (fig. 1 A)

Dans la zone céphalo-pédieuse, la paroi des sporocystes transplantés est le siège d'une intense activité mitotique qui est à l'origine de la différenciation d'une nouvelle génération larvaire. Les jeunes larves formées au sein des sporocystes transplantés sont en tous points morphologiquement semblables à celles issues du sporocyste primaire $(\mathrm{SpI})$, elles en possèdent les caractères différentiels : aspect vermiforme, tégument bien différencié et absence de " primordial gland cells ".

Les nouvelles générations de sporocystes-fils essaiment vers les différents organes du Mollusque (manteau, pied et principalement glande digestive et génitale). Les sporocystes répliqués après transplantation ne vont pas tous suivre la même évolution. Comme dans le cas des infestations naturelles (Combes et al., 1983), on observe une dualité morphogénétique : une partie des sporocystes, majoritaire, se réplique sans cercariogenèse préalable assurant ainsi une colonisation rapide de l'hôte ; l'autre partie, minoritaire, s'oriente directement vers la cercariogenèse.

\section{$\mathrm{c}-79$ jours}

A ce stade, l'émission des cercaires a commencé. Il subsiste toujours dans la zone céphalo-pédieuse des formes larvaires correspondant à des sporocystes répliqués soit en phase de sporocystogenèse soit en phase de cercariogenèse avec des cercaires entièrement différenciées. Néanmoins, il est fréquent d'observer dans cette zone des cercaires en voie de dégénérescence.

La glande digestive est envahie par des sporocystes répliqués dont la lumière renferme des cercaires en cours et en fin de maturation. Nous avons montré que la sortie des cercaires se faisait essentiellement par effraction à travers la paroi du sporocyste, ce qui confirme les observations de Lengy (1962). Cette action mécanique engendre une infiltration des hémocytes de l'hôte à l'intérieur du sporocyste entrainant une lyse des cercaires et de la paroi du sporocyste.

\section{d -118 jours (fig. 1 B)}

Les sporocystes engagés dans les deux modes de différenciation décrits ci-dessus sont toujours présents dans la sphère céphalo-pédieuse.

La glande digestive est entièrement et principalement colonisée par des sporocystes cercariogènes. Le processus d'infiltration hémocytaire suivi de dégénérescence cercarienne s'intensifie chez les sporocystes en fin de production. Il subsiste dans la glande digestive des sporocystes sporocystogènes dont l'origine peut être double : - il s'agirait soit de sporocystes provenant du greffon resté actif dans la zone céphalo-pédieuse et qui se sont d'emblée orientés vers la sporocystogenèse ;

- soit d'une reconversion in situ dans la glande digestive après dédifférenciation de sporocystes cercariogènes.

\section{Production cercarienne et infectivité des cercaires}

L'émission cercarienne a été testée à partir du $20^{\mathrm{e}}$ jour suivant la transplantation. Les premières cercaires ont été émises selon les Mollusques entre le $36^{\mathrm{e}}$ et le $40^{\mathrm{e}}$ jour. Le taux d'infestation a été de $71 \%$. 

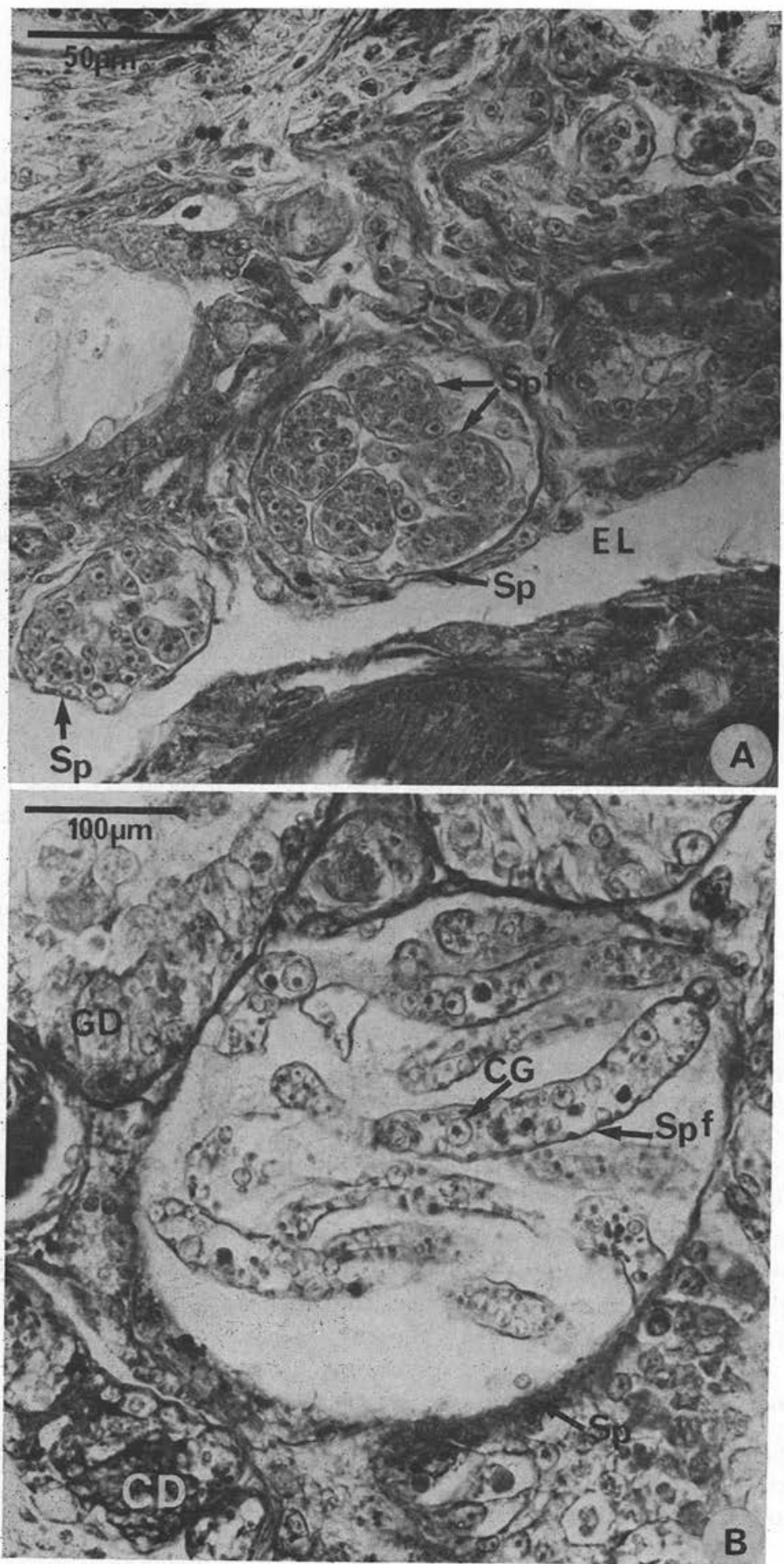
Notre étude sur la production cercarienne a porté sur 7 Mollusques. Pour l'ensemble de la durée de notre expérience nous avons observé que la production de chacun d'eux obéissait à une dynamique très proche : celle-ci peut se décomposer en 3 phases d'amplitude différente allant dans le sens d'une augmentation de la quantité de cercaires émises. La production cercarienne représentée sur la figure 2, correspondant à la production des deux Mollusques ayant présenté la longévité la plus importante, illustre de façon très significative ces trois phases d'émission :

- phase I de faible production $(49 \mathrm{C} / \mathrm{J} / \mathrm{M}$ en moyenne pour les 2 Mollusques) qui dure environ 3 semaines ;

- phase II de forte production ( $454 \mathrm{C} / \mathrm{J} / \mathrm{M}$ en moyenne pour les 2 Mollusques) au cours de laquelle la quantité de cercaires produites est 9 fois plus forte qu'au cours de la phase I; elle dure 5 semaines environ ;

— phase III de production maximale (1045 C/J/M pour les 2 Mollusques), 2 fois plus forte que la phase II et qui dure 3 semaines.

La dernière phase est suivie par une chute brutale de la production $(70 \mathrm{C} / \mathrm{J} / \mathrm{M})$; la mort du Mollusque survient au cours de cette période.

$\mathrm{Au}$ plan quantitatif, la production cercarienne observée chez les Mollusques transplantés est très voisine de celle que nous avons nous-mêmes obtenu à partir de Mollusques infestés par voie miracidiale.

Pour des raisons d'ordre matériel, nous n'avons pu mener qu'un travail préliminaire sur l'infectivité des cercaires. Néanmoins, nos résultats montrent que le pourcentage de développement des cercaires en adultes n'est pas très différent de celui noté à partir de cercaires produites par des Mollusques infestés par voie miracidiale.

\section{Discussion}

Les résultats que nous avons obtenus s'accordent avec les données concernant l'évolution intramolluscale des sporocystes fils transplantés de $S$. mansoni et de S. haematobium. Notre travail rend compte de la même façon d'une dédifférenciation des sporocystes transplantés et de leur différenciation en sporocystes sporocystogènes producteurs d'une génération additionnelle des sporocystes fils.

FIG. I. - Évolution histologique du greffon

(sporocyste-fils de $S$. bovis) après transplantation microchirurgicale chez B. truncatus.

A. Stade I 8 jours : greffon localisé dans l'espace lacunaire céphalo-pédieux (E.L.) du Mollusque. Les sporocystes transplantés (Sp) ont subi la dédifférenciation les conduisant à la production de sporocystes-fils (Spf) par réplication.

B. Stade 118 jours: on peut observer côte à côte dans la glande digestive (G. D) des sporocystes cercariogènes offrant souvent des images de dégénérescence de cercaires (C.D) et des sporocystes sporocystogènes $(\mathrm{Sp})$ en phase de réplication

Spf : sporocyste fils répliqué - C.G : cellules germinatives. 

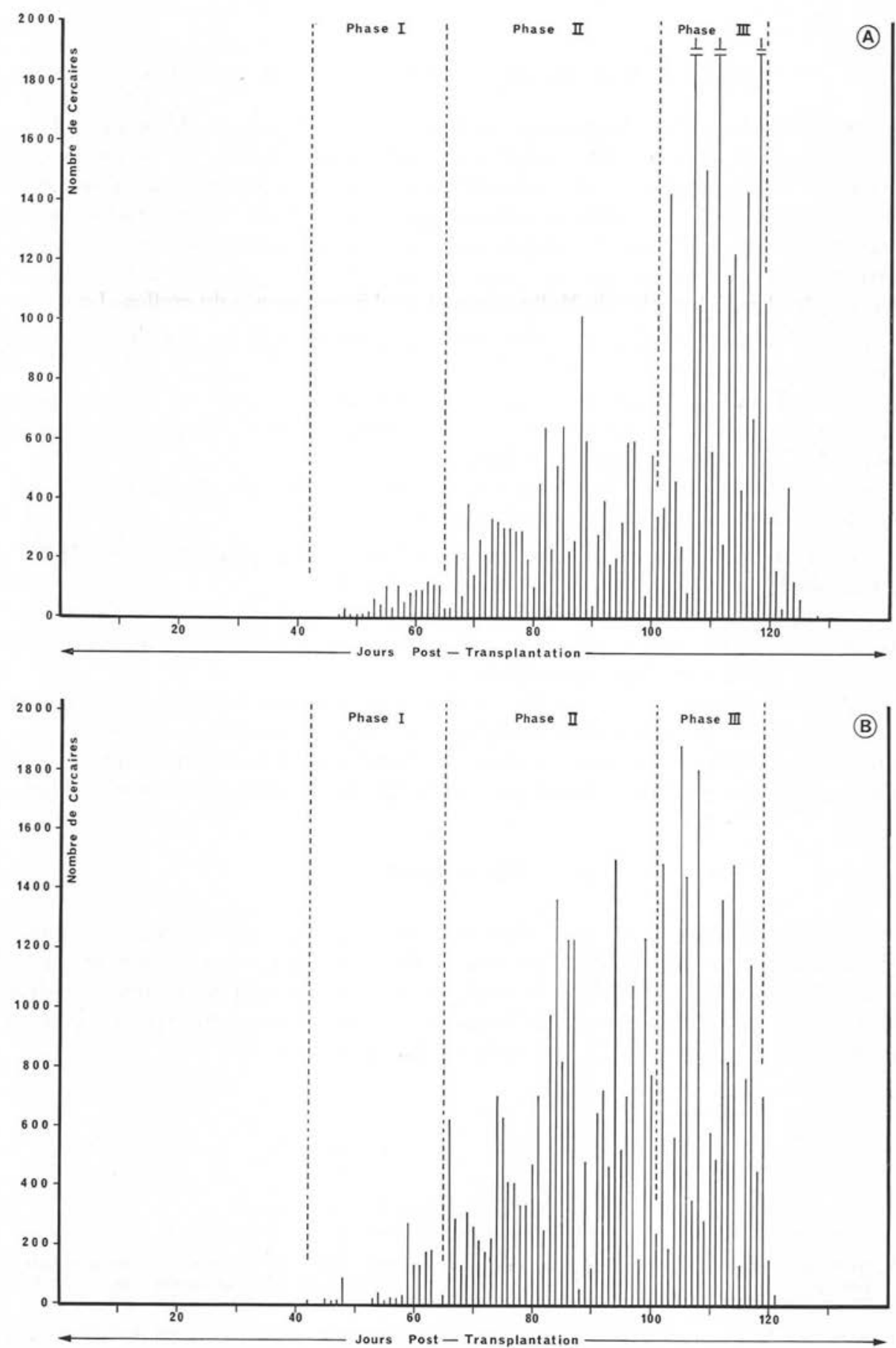

FIG. 2. - Production cercarienne journalière de $S$. bovis (Soudan) par 2 B. truncatus (Tunisie) après transplantation microchirurgicale de sporocystes.

Les productions journalières du Mollusque A correspondant au ro $7^{\mathrm{e}}$ jour, II $\mathrm{I}^{\mathrm{e}}$ jour et au Ir $8^{\text {e }}$ jour sont respectivement 2320,2800 et 2064 cercaires. 
Néanmoins, chez $S$. bovis, la période d'invasion parasitaire post-transplantation offre des modalités différentes de celles décrites chez $S$. mansoni et $S$. haematobium. La sphère céphalo-pédieuse constitue en effet chez cette espèce pendant plus de quatre mois le siège d'une intense multiplication asexuée du parasite. A la différence de ce qui est connu de l'évolution de $S$. mansoni et $S$. haematobium après transplantation, on note ici qu'une fraction de la population des sporocystes répliqués n'émigre pas vers la glande digestive du Mollusque mais se développe près du greffon. La grande majorité des sporocystes répliqués restés in situ vont être à leur tour intéressés par un processus de sporocystogenèse qui aura pour conséquence d'assurer une exploitation optimale de la capacité biotique du Mollusque. Il convient de souligner que ce mode de reproduction diffère beaucoup de celui observé après infestation par voie miracidiale (Combes et al., 1983). Par contre, il offre beaucoup de points communs avec le modèle de multiplication asexuée intrapédieuse récemment découvert dans les conditions naturelles chez S. haematobium (Jourdane, 1983).

Concernant la dynamique de la production cercarienne, nos données révèlent que celle-ci serait au moins en partie en relation avec la dynamique intramolluscale des populations de sporocystes.

Au cours des 35 premiers jours, l'absence d'émission cercarienne s'explique très facilement par le fait que cette période correspond à la période nécessaire à la reconversion morphogénétique des sporocystes transplantés et à la mise en place d'une première vague de sporocystes cercariogènes.

La phase de faible productivité cercarienne observée pendant les 3 premières semaines d'émission reflèterait de façon fidèle les particularités du développement intramolluscal après transplantation : nous avons en effet signalé que seule une fraction de la population de sporocystes issus de la réplication du matériel transplanté s'engageait dans la voie de la cercariogenèse, ce qui explique la faible émission cercarienne observée.

L'accroissement de la production cercarienne au cours des phases II et III est liée à l'augmentation corrélative de la population de sporocystes cercariogènes issus de la réplication des sporocystes fils.

Quant à la chute brutale de la production après la phase III, elle est certainement consécutive à l'emplification des processus de dégénérescence des sporocystes liés aux infiltrations hémocytaires de plus en plus importantes.

La réussite de la transplantation de $S$. bovis chez B. truncatus permet d'envisager de façon positive la possibilité de clonage de l'espèce. Il serait en effet surprenant, après les résultats obtenus dans ce domaine pour S. mansoni et S. haematobium, que la réussite d'une première transplantation ne soit pas suivie d'un succès des transplantations en série et de manière indéfinie.

Les différentes données sur la production cercarienne et sur l'infectivité des cercaires après une première transplantation sont de nature à faire adopter le clonage comme une technique de routine dans la maintenance au laboratoire de $S$. bovis. 


\section{BIBLIOGRAPHIE}

Combes C., Touassem R., Mouahid A. : Mise en évidence de l'intervention de sporocystes réplicateurs dans le cycle biologique de Schistosoma bovis (Sonsino, 1876) Blanchard, I895. Bull. Soc. Fr. Parasitol., 1983, 1, 27-30.

Duwall R. H., DewitT W. B. : An improved perfusion technique for recovering adult schistosomes from laboratory animals. Am. J. Trop. Med. Hyg., I967, I6, 483-486.

Jourdane J. : Mise en évidence d'un processus original de la reproduction asexuée chez Schistosoma haematobium. C. R. Acad. Sci. Paris, 1983, 296, 419-424.

Jourdane J., Kechemir N., Combes C. : Mise en évidence d'une réplication de sporocystes fils de Schistosoma haematobium après transplantation microchirurgicale chez Bulinus truncatus. C.R. Acad. Sci. Paris, 1981, 293, 531-533.

Jourdane J., Theron A. : Schistosoma mansoni : cloning by microsurgical transplantation of sporocysts. Exp. Parasitol., I980, so, 349-357.

Jourdane J., Theron A., Combes C. : Demonstration of several sporocysts generations as a normal pattern of reproduction of Schistosoma mansoni. Acta Tropica, 1980, 37, 177-182.

Kechemir N., Combes C. : Développement du Trématode Schistosoma haematobium après transplantation microchirurgicale chez le Gastéropode Planorbarius metidjensis. C.R. Acad. Sci. Paris, $1982,295,505-508$.

Kechemir N., Theron A. : Existence of replicating sporocysts in the development cycle of Schistosoma haematobium. J. Parasitol., 1980, 66, 1068-1070.

LENGY J. : Studies on Schistosoma bovis (Sonsino, I876) in Israel. I - Larval stages from egg to cercaria. Bull. Res. Counc. Isvael, I962, I0, I-36.

Theron A. : Le compartiment cercaire dans le cycle de Schistosoma mansoni Sambon, 1907. Écologie de la transmission bilharzienne en Guadeloupe. Thèse d'État, Perpignan, 1982. 506 p.

Cette recherche a reçu le support financier de l'Edna Mac Connel Clark Foundation et du Programme Spécial P.N.U.D., Banque Mondiale O.M.S. de Recherche et de Formation concernant les maladies tropicales. 